\title{
Effects of convective conditions and chemical reaction on peristaltic flow of Eyring-Powell fluid
}

\author{
T. Hayat ${ }^{\mathrm{a}, \mathrm{b}}$, Anum Tanveer ${ }^{\mathrm{a}}$, Humaira Yasminin ${ }^{\mathrm{a}, *}$ and A. Alsaedi ${ }^{\mathrm{b}}$ \\ ${ }^{a}$ Department of Mathematics, Quaid-I-Azam University, Islamabad, Pakistan \\ ${ }^{\mathrm{b}}$ Department of Mathematics, Faculty of Science, King Abdulaziz University, Jeddah, Saudi Arabia
}

\begin{abstract}
This paper addresses the peristaltic flow of Eyring-Powell fluid in a symmetric channel with convective conditions. The Soret and Dufour effects are considered. Impact of first order chemical reaction is seen. The channel walls are of compliant nature. Long wavelength and low Reynolds number concepts are implemented. Resulting problems are solved for the stream function, temperature and concentration. Graphical results are presented and discussed in detail for various pertinent parameters.
\end{abstract}

Keywords: Eyring-powell fluid, convective conditions, soret and dufour effects, chemical reaction

\section{Introduction}

The fluid transport by peristalsis has vital importance in biology, medicine and industry. Principle of peristalsis in fact represents travelling contraction wave along a tube like structure. Such mechanism occurs naturally as a mean of pumping physiological fluids from one place in the body to another. Some electro-chemical reactions are held responsible for this phenomenon. No doubt the peristalsis is an inherent property of many of the smooth muscle tubes such as bile duct, ureter etc. Esophagus is a flexible muscular tube and peristalsis here occurs when muscles in the esophagus wall work in a coordinated manner to push food and liquids down into the stomach. The gastrointestinal tract is also surrounded by innervated smooth muscle layers. The contraction of such muscle layers mix the contents of tract and thus responsible for food movement in an appropriate direction. The worms employ peristalsis for locomotion. The

*Corresponding author: Humaira Yasmin, Department of Mathematics, Quaid-I-Azam University 45320, Islamabad 44000, Pakistan. Tel.: +92 51 90642172; E-mail: qau2011@gmail.com. phenomenon of peristalsis through peristaltic pumps is useful in the industries such as chemical industry, ceramic and porcelain industry, building industry, food and paper, heart-lung machine etc. In nuclear industry such activity is employed not to contaminate the outside environment. The majority of the aforementioned applications involve the viscoelastic fluids and solid wall mechanism of the flexible tube in predicting wall deformation. Viscoelastic fluids are non-Newtonian and possess both the viscous and elastic properties (see [1-6]). Most of the biological fluids such as blood (at low shear rate), chyme and food bolus are viscoelastic in nature. Motivated by such considerations few related investigations on the topic may be represented through the studies [7-13]. Further, the significance of convective heat exchange with peristalsis cannot be under estimated for example in translocation of water in tall trees, dynamics of lakes, lubrication and drying technologies, diffusion of nutrients out of blood, oxygenation, hemodialysis and nuclear reactors. In fact heat transfer in biological tissues involve processes like heat conduction in tissues, heat convection due to blood flow through the pore of tissues, as well as radiation heat transfer between surface and its environment. 
Heat transfer between a solid boundary and static fluid takes place due to conduction purely. Such type of problems gives rise to boundary conditions through Fourier's law of heat conduction. However, the transfer of heat between solid boundary and a moving fluid is due to both conduction and convection. The boundary condition in such case is because of Fourier's law of heat conduction and the Newton's law of cooling. This type of boundary condition is called the convective type. The convective conditions have key role for maintaining a healthy building in view of fresh air ventilation and membrane-based air-to-air heat mass exchangers. These are also important in heat transfer processes like hemodialysis, cancer therapy etc. Further, the significance of convective heat exchange with peristalsis cannot be under estimated for example in translocation of water in tall trees, dynamics of lakes, lubrication and drying technologies, diffusion of nutrients out of blood, oxygenation and nuclear reactors. The simultaneous effects of heat and mass transfer are of great interest in chemically distillatory processes, formation and dispersion of fog, environmental pollution, food processing, lubrication technologies and several others. Motivated by such facts some recent researchers put forward the analysis of peristaltic flows in the presence of heat/mass transfer [14-25].

Existing information on peristalsis witnesses that no proper attention is focused to the peristaltic flows of viscoelastic fluids in the presence of Soret and Dufour effects. The objective of present investigation is to fill this void. Hence the Eyring-Powell fluid is used to describe the viscoelastic properties [26-28]. This fluid model can describe certain advantages over the other non-Newtonian fluid models. Firstly it is deduced from kinetic theory of liquids rather than empirical relation. Secondly it correctly reduces to Newtonian behavior for low and high shear rates. Mathematical modelling here is made in the presence of chemical reaction and Soret and Dufour effects. The compliant wall properties of channel walls are taken into account. Analysis of nonlinear system is analyzed through the series solution. Results for physical quantities of interest are obtained. Impact reflecting the influence of pertinent variables is pointed out in detail.

\section{Problem formulation and flow equations}

Consider the flow of an incompressible EyringPowell fluid in two-dimensional horizontal channel of

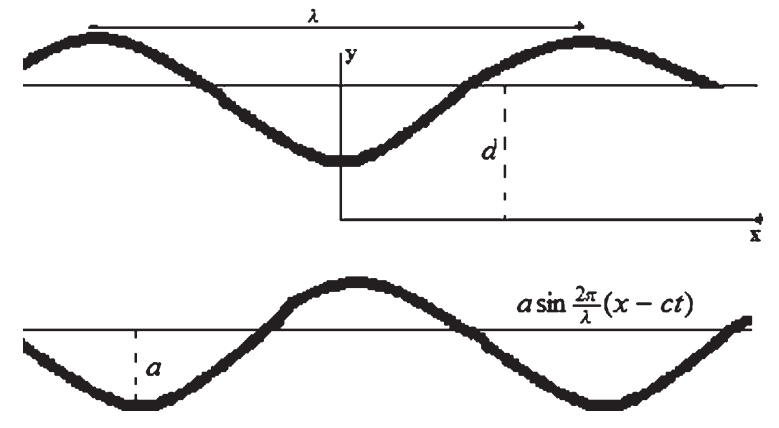

Fig. 1. Geometry of the problem.

width $2 d$. We consider Cartesian coordinates in such a manner that wave propagate parallel to $x$-direction. Here $y$-axis is taken transverse to the $x$-axis. The walls of channel are flexible subject to viscous damping effects. The waves are considered sinusoidal with wavelength $\lambda$. Such waves travel with constant speed $c$ along the channel walls. The wall surface is described by the expression

$$
y= \pm \eta(x, t)=d+a \sin \frac{2 \pi}{\lambda}(x-c t),
$$

where $c$ is the wave speed, $a$ the wave amplitude, $\lambda$ the wavelength, $2 d$ the width of symmetric channel, $t$ the time, $\eta$ displacement of upper wall and $-\eta$ displacement of lower wall. For incompressible fluid the continuity equation is given by

$$
\operatorname{div} \mathbf{V}=0
$$

The momentum equation is

$$
\rho \frac{d \mathbf{V}}{d \bar{t}}=-\nabla p+\operatorname{div} \mathbf{S} .
$$

The equations for energy and mass with Soret and Dufour effects are as follows:

$$
\begin{aligned}
\rho c_{p} \frac{d T}{d \bar{t}} & =k \nabla^{2} T+\frac{D K_{T}}{c_{s}}\left(\nabla^{2} C\right), \\
\frac{d C}{d \bar{t}} & =D \nabla^{2} C+\frac{D K_{T}}{T_{m}}\left(\nabla^{2} T\right)-k_{1}\left(C-C_{0}\right),
\end{aligned}
$$

where the extra stress tensor for Eyring-Powell fluid is given by [5]:

$$
S_{i j}=\mu \frac{\partial u_{i}}{\partial x_{j}}+\frac{1}{\beta} \sinh ^{-1}\left(\frac{1}{c_{1}} \frac{\partial u_{i}}{\partial x_{j}}\right), \quad i, j=1,2
$$


where $u_{1}=u, u_{2}=v, x_{1}=x$ and $x_{2}=y$. Also

$$
\begin{aligned}
\sinh ^{-1}\left(\frac{1}{c_{1}} \frac{\partial u_{i}}{\partial x_{j}}\right) & =\frac{1}{c_{1}} \frac{\partial u_{i}}{\partial x_{j}}-\frac{1}{6}\left(\frac{1}{c_{1}} \frac{\partial u_{i}}{\partial x_{j}}\right)^{3}, \\
\left|\frac{1}{c_{1}} \frac{\partial u_{i}}{\partial x_{j}}\right| & \ll 1 .
\end{aligned}
$$

Equation (6) in component form yields

$$
\begin{aligned}
& S_{x x}=\mu \frac{\partial u}{\partial x}+\frac{1}{\beta}\left[\frac{1}{c_{1}} \frac{\partial u}{\partial x}-\frac{1}{6 c_{1}^{3}}\left(\frac{\partial u}{\partial x}\right)^{3}\right], \\
& S_{x y}=\mu \frac{\partial u}{\partial y}+\frac{1}{\beta}\left[\frac{1}{c_{1}} \frac{\partial u}{\partial y}-\frac{1}{6 c_{1}^{3}}\left(\frac{\partial u}{\partial y}\right)^{3}\right], \\
& S_{y x}=\mu \frac{\partial v}{\partial x}+\frac{1}{\beta}\left[\frac{1}{c_{1}} \frac{\partial v}{\partial x}-\frac{1}{6 c_{1}^{3}}\left(\frac{\partial v}{\partial x}\right)^{3}\right], \\
& S_{y y}=\mu \frac{\partial v}{\partial y}+\frac{1}{\beta}\left[\frac{1}{c_{1}} \frac{\partial v}{\partial y}-\frac{1}{6 c_{1}^{3}}\left(\frac{\partial v}{\partial y}\right)^{3}\right],
\end{aligned}
$$

in which $\frac{d}{d \bar{t}}$ is material time derivative, $\mathbf{V}=[u, v]$ the velocity, $u, v$ the components of $\mathbf{V}$ in $x$ and $y$ directions respectively, $\rho$ density of fluid, $\mu$ the dynamic fluid viscosity, $v$ the kinematic viscosity, $T$ the fluid temperature, $C$ the mass concentration, $T_{0}$ the temperature at the walls and $C_{0}, C_{1}$ the concentration at the lower and upper walls respectively, $T_{m}$ the mean temperature of fluid, $D$ the coefficient of mass diffusivity, $c_{p}$ the specific heat at constant pressure, $k$ the thermal conductivity, $k_{1}$ the chemical reaction parameter, $\beta$ and $c_{1}$ the material fluid parameters of Eyring-Powell fluid, $c_{s}$ the concentration susceptibility, $K_{T}$ the thermal diffusion ratio, $\mathbf{S}$ the extra stress tensor and $\nabla^{2}=\left(\frac{\partial^{2}}{\partial x^{2}}+\frac{\partial^{2}}{\partial y^{2}}\right)$.

The exchange of heat at the walls is given by

$$
\begin{aligned}
& k \frac{\partial T}{\partial y}=-h_{1}\left(T-T_{0}\right) \text { at } y=\eta, \\
& k \frac{\partial T}{\partial y}=-h_{2}\left(T_{0}-T\right) \text { at } y=-\eta .
\end{aligned}
$$

Here $h_{1}$ and $h_{2}$ indicate the heat transfer coefficients at the upper and lower walls respectively.

The no-slip condition at the walls is represented by the following expressions

$$
u=0, v= \pm \eta_{t} \text { at } y= \pm \eta .
$$

Further the concentration conditions are given by

$$
C=\left\{\begin{array}{l}
C_{1} \\
C_{0}
\end{array}\right\}, \quad \text { at } y= \pm \eta .
$$

Due to continuity of stress the pressure exerted by the walls on the fluid is equal and opposite to pressure exerted by the fluid on the walls. Also transverse displacements of walls are equal to corresponding $y$ displacements of fluid at instantaneous positions. Now continuity of stress and deformation condition collectively give the no separation condition at the boundaries. Mathematically one can thus write

$$
\begin{aligned}
& {\left[-\tau \frac{\partial^{3}}{\partial x^{3}}+m_{1} \frac{\partial^{3}}{\partial x \partial t^{2}}+d^{\prime} \frac{\partial^{2}}{\partial t \partial x}\right] \eta} \\
& \quad=-\rho \frac{d u}{d t}+\frac{\partial S_{x x}}{\partial x}+\frac{\partial S_{x y}}{\partial y} \text { at } y= \pm \eta
\end{aligned}
$$

where $\tau$ is the elastic tension in the membrane, $m_{1}$ the mass per unit area and $d^{\prime}$ the coefficient of viscous damping.

The scaler equations through Eq. (3) are

$$
\begin{aligned}
& \rho \frac{d u}{d t}=-\frac{\partial p}{\partial x}+\frac{\partial S_{x x}}{\partial x}+\frac{\partial S_{x y}}{\partial y}, \\
& \rho \frac{d v}{d t}=-\frac{\partial p}{\partial y}+\frac{\partial S_{y x}}{\partial x}+\frac{\partial S_{y y}}{\partial y} .
\end{aligned}
$$

Performing $\frac{\partial}{\partial y}(17)-\frac{\partial}{\partial x}$ (18) we get

$\rho \frac{d}{d \bar{t}}\left(\frac{\partial u}{\partial y}-\frac{\partial v}{\partial x}\right)=\frac{\partial S_{x x}}{\partial x}-\frac{\partial^{2} S_{y x}}{\partial x^{2}}+\frac{\partial^{2} S_{x y}}{\partial y^{2}}-\frac{\partial^{2} S_{y y}}{\partial x \partial y}$

Introducing the stream function $\psi(x, y, t)$ and defining the following dimensionless variables:

$$
u=\frac{\partial \psi}{\partial y}, v=-\frac{\partial \psi}{\partial x}
$$

$$
\begin{gathered}
\psi^{*}=\frac{\psi}{c d}, x^{*}=\frac{x}{\lambda}, y^{*}=\frac{y}{d}, t^{*}=\frac{c t}{\lambda}, \\
\eta^{*}=\frac{\eta}{d}, \gamma=k_{1} \frac{d^{2}}{v}, \theta=\frac{T-T_{0}}{T_{0}}, \\
\phi=\frac{C-C_{0}}{C_{1}-C_{0}}, S_{x x}^{*}=\frac{d}{\mu c} S_{x x}, S_{x y}^{*}=\frac{d}{\mu c} S_{x y}, \\
S_{y x}^{*}=\frac{d}{\mu c} S_{y x}, S_{y y}^{*}=\frac{d}{\mu c} S_{y y},
\end{gathered}
$$

we arrive at 


$$
\begin{aligned}
& S_{x x}^{*}=\delta \psi_{y x}^{*}(1+M)-\delta^{3} K \psi_{y x}^{* 3}, \\
& S_{x y}^{*}=\psi_{y y}^{*}(1+M)-K \psi_{y y}^{* 3}, \\
& S_{y x}^{*}=-\delta^{2} \psi_{x x}^{*}(1+M)+\delta^{6} K \psi_{x x}^{* 3}, \\
& S_{y y}^{*}=-\delta \psi_{y x}^{*}(1+M)+\delta^{3} K \psi_{y x}^{* 3},
\end{aligned}
$$

and Eqs. (4), (5) and (19) yield

$$
\begin{aligned}
& \delta \operatorname{Re}\left[\frac{d}{d t}\left(\frac{\partial^{2} \psi}{\partial y^{2}}+\delta^{2} \frac{\partial^{2} \psi}{\partial x^{2}}\right)\right] \\
& =\delta \frac{\partial^{2} S_{x x}}{\partial y \partial x}+\delta^{2} \frac{\partial^{2} S_{y x}}{\partial x^{2}}+\frac{\partial^{2} S_{x y}}{\partial y^{2}}-\delta^{2} \frac{\partial^{2} S_{y y}}{\partial x \partial y},
\end{aligned}
$$

$\delta \operatorname{Pr} \operatorname{Re} \frac{d}{d t} \theta$

$$
\begin{gathered}
=\delta^{2} \frac{\partial^{2} \theta}{\partial x^{2}}+\frac{\partial^{2} \theta}{\partial y^{2}}+\operatorname{Pr} D u\left[\delta^{2} \frac{\partial^{2} \phi}{\partial x^{2}}+\frac{\partial^{2} \phi}{\partial y^{2}}\right], \\
\frac{1}{S c} \frac{\partial^{2} \phi}{\partial y^{2}}+S r \frac{\partial^{2} \theta}{\partial y^{2}}-\gamma \phi=0,
\end{gathered}
$$

with the conditions

$$
\begin{aligned}
& \eta=1+\epsilon \sin 2 \pi(x-t), \\
& \theta_{y}+\gamma_{1} \theta=0 \text { at } y=\eta, \\
& \theta_{y}-\gamma_{2} \theta=0 \text { at } y=-\eta, \\
& \phi=\left\{\begin{array}{l}
1 \\
0
\end{array}\right\} \text { at } y= \pm \eta, \\
& \psi_{y}=0 \text { at } y= \pm \eta, \\
& {\left[E_{1} \frac{\partial^{3}}{\partial x^{3}}+E_{2} \frac{\partial^{3}}{\partial x \partial t^{2}}+E_{3} \frac{\partial^{2}}{\partial x \partial t}\right] \eta } \\
&=\delta \frac{\partial}{\partial x} S_{x x}+\frac{\partial}{\partial y} S_{x y} \text { at } y= \pm \eta .
\end{aligned}
$$

In above equations asterisks have been omitted for simplicity and the dimensionless wave number $\delta$, the Reynolds number Re, the Prandtl number $\mathrm{Pr}$, the Eckert number $E$, the amplitude ratio $\epsilon$, the chemical reaction parameter $\gamma$, the Eckert number $E$, the non dimensional elasticity parameters $E_{1}, E_{2}, E_{3}$, the Schmidt number $S c$, the Soret number $S r$, the Dufour number $D u$, the Biot numbers $\gamma_{1}$ and $\gamma_{2}$ and the fluid parameters $M$ and $K$ are given by the following definitions:

$$
\begin{aligned}
& \delta=\frac{d}{\lambda}, \operatorname{Re}=\frac{c d}{v}, \operatorname{Pr}=\frac{\mu c_{p}}{k}, \\
& \epsilon=\frac{a}{d}, \gamma=\frac{k_{1} d^{2}}{v}, E=\frac{c^{2}}{T_{0} c_{p}}, \\
& E_{1}=-\frac{\tau d^{3}}{\lambda^{3} \mu c}, E_{2}=\frac{m_{1} c d^{3}}{\lambda^{3} \mu}, \\
& E_{3}=\frac{d^{3} d^{\prime}}{\mu \lambda^{2}}, S c=\frac{\mu}{\rho D}, M=\frac{1}{\mu \beta c_{1}}, \\
& K=\frac{M c^{2}}{6 d^{2} c_{1}^{2}}, D u=\frac{D K_{T}\left(C_{1}-C_{0}\right)}{c_{s} c_{p} v T_{0}}, \\
& S r=\frac{D K_{T} T_{0}}{v T_{m}\left(C_{1}-C_{0}\right)}, \gamma_{1}=\frac{h_{1} d}{k}, \\
& \gamma_{2}=\frac{h_{2} d}{k} .
\end{aligned}
$$

Long wavelength and low Reynolds number considerations lead to the following expressions

$$
\begin{gathered}
(1+M) \frac{\partial^{4} \psi}{\partial y^{4}}-6 K \frac{\partial^{2} \psi}{\partial y^{2}}\left(\frac{\partial^{3} \psi}{\partial y^{3}}\right)^{2} \\
-3 K\left(\frac{\partial^{2} \psi}{\partial y^{2}}\right)^{2} \frac{\partial^{4} \psi}{\partial y^{4}}=0, \\
\frac{\partial^{2} \theta}{\partial y^{2}}+\operatorname{Pr} D u \frac{\partial^{2} \phi}{\partial y^{2}}=0, \\
S r \frac{\partial^{2} \theta}{\partial y^{2}}+\frac{1}{S c} \frac{\partial^{2} \phi}{\partial y^{2}}-\gamma \phi=0, \\
\psi_{y}=0 \quad \text { at } y= \pm \eta, \\
\theta_{y}+\gamma_{1} \theta=0 \text { at } y=\eta \\
\theta_{y}-\gamma_{2} \theta=0 \text { at } y=-\eta, \\
\phi=\left\{\begin{array}{l}
1 \\
0
\end{array}\right\} \text { at } y= \pm \eta,
\end{gathered}
$$

$$
\begin{aligned}
& {\left[E_{1} \frac{\partial^{3}}{\partial x^{3}}+E_{2} \frac{\partial^{3}}{\partial x \partial t^{2}}+E_{3} \frac{\partial^{2}}{\partial x \partial t}\right] \eta} \\
& =(1+M) \frac{\partial^{3} \psi}{\partial y^{3}}-3 K\left(\frac{\partial^{2} \psi}{\partial y^{2}}\right)^{2} \frac{\partial^{3} \psi}{\partial y^{3}} \text { at } y= \pm \eta
\end{aligned}
$$




\section{Method of solution}

The exact solutions of resulting Equations (38-39) with conditions (41-42) are found as:

\subsection{First order system and solution}

At this order we have

$$
\begin{aligned}
\theta & =\frac{1}{2} \times D u \operatorname{Pr}\left(\begin{array}{c}
L_{4}+L_{2} y \operatorname{csch}\left(L_{2} \eta\right)+\operatorname{sech}\left(L_{2} \eta\right)-\cosh \left(L_{2} y\right) \operatorname{sech}\left(L_{2} \eta\right) \\
-\operatorname{csch}\left(L_{2} \eta\right) \sinh \left(L_{2} y\right)-L_{5} y
\end{array}\right), \\
\phi & =\operatorname{csch}\left(2 L_{2} \eta\right) \sinh \left(L_{2}(y+\eta)\right) .
\end{aligned}
$$

Heat transfer coefficient at the wall is given by

$$
\begin{aligned}
Z & =\eta_{x} \theta_{y}(\eta) \\
& =\frac{\eta_{x} D u \operatorname{Pr}\left[-L_{2} \operatorname{coth}\left(L_{2} \eta\right)+L_{2} \operatorname{csch}\left(L_{2} \eta\right)-L_{5}-L_{2} \tanh \left(L_{2} \eta\right)\right]}{2} .
\end{aligned}
$$

Since the resulting equation (37) is non-linear. For arbitrary values of parameters involving in this equation, the closed form solution seems very difficult to obtain. Attention is hence focused to the perturbation solution for small fluid parameter $K$. For that we expand stream function $\psi$ in series form as:

$$
\psi=\psi_{0}+K \psi_{1}+O\left(K^{2}\right)
$$

\subsection{Zeroth order system and solution}

The resulting equation at the zeroth order is given by

$$
\begin{aligned}
& \frac{\partial^{4} \psi_{0}}{\partial y^{4}}=0, \\
& \psi_{0 y}=0, \quad \text { at } y= \pm \eta \\
& {\left[E_{1} \frac{\partial^{3}}{\partial x^{3}}+E_{2} \frac{\partial^{3}}{\partial x \partial t^{2}}+E_{3} \frac{\partial^{2}}{\partial x \partial t}\right] \eta} \\
& \quad=(1+M) \psi_{0 y y y} \text { at } y= \pm \eta .
\end{aligned}
$$

The solutions of Eq. (45) subject to the boundary conditions $(46-47)$ is

$$
\psi_{0}=y\left(\frac{L y^{2}}{3}-L_{1}\right),
$$

It should be pointed out that solution expressions for zeroth order system correspond to the results of viscous fluid [19] in absence of Soret and Dufour effects for prescribed surface temperature.

$$
\begin{aligned}
& (1+M) \frac{\partial^{4} \psi_{1}}{\partial y^{4}}-6 \frac{\partial^{2} \psi_{0}}{\partial y^{2}}\left(\frac{\partial^{3} \psi_{0}}{\partial y^{3}}\right)^{2} \\
& \quad-3\left(\frac{\partial^{2} \psi_{0}}{\partial y^{2}}\right)^{2} \frac{\partial^{4} \psi_{0}}{\partial y^{4}}=0 \\
& \psi_{1 y}=0, \text { at } y= \pm \eta \\
& (1+M) \frac{\partial^{3} \psi_{1}}{\partial y^{3}}-3\left(\frac{\partial^{2} \psi_{0}}{\partial y^{2}}\right)^{2} \frac{\partial^{3} \psi_{0}}{\partial y^{3}}=0 \text { at } y= \pm \eta
\end{aligned}
$$

Solving the resulting equations (49) and then applying the corresponding boundary conditions we get the solutions for $\psi_{1}$ in the form given below:

$$
\psi_{1}=A y\left(\eta^{4}-\frac{y^{4}}{5}\right)
$$

where

$$
\begin{aligned}
& L=\frac{2 \pi^{2} \epsilon\left(-2\left(E_{1}+E_{2}\right) \pi \cos 2 \pi(x-t)+E_{3} \sin 2 \pi(x-t)\right)}{(1+M)}, \\
& L_{1}=L(1+\epsilon \sin 2 \pi(x-t))^{2}, \\
& L_{2}=\frac{\sqrt{S c \gamma}}{\sqrt{1-D u \operatorname{Pr} S c S r}}, \\
& L_{3}=(-1+D u \operatorname{Pr} S c S r), \\
& L_{4}=\frac{2}{L_{3}\left(\gamma_{1}+\gamma_{2}+2 \gamma_{1} \gamma_{2} \eta\right)}
\end{aligned}
$$




$$
\begin{aligned}
& \times\left(\begin{array}{c}
-\sqrt{S c \gamma L_{3}}\left(1+\gamma_{2} \eta\right) \operatorname{coth}\left(2 L_{2} \eta\right)+\sqrt{-S c \gamma L_{3}}\left(1+\gamma_{1} \eta\right) \operatorname{csch}\left(2 L_{2} \eta\right) \\
+\frac{1}{2} L_{3}\left\{2 \gamma_{1}+2 \gamma_{1} \gamma_{2} \eta-\left(\gamma_{1}+\gamma_{2}+2 \gamma_{1} \gamma_{2} \eta\right) \operatorname{sech}\left(L_{2} \eta\right)\right\}
\end{array}\right), \\
L_{5}= & \frac{2 \sqrt{\gamma-D u \operatorname{Pr} S c S r \gamma} \operatorname{csch}\left(2 L_{2} \eta\right)}{L_{3} \gamma\left(\gamma_{1}+\gamma_{2}+2 \gamma_{1} \gamma_{2} \eta\right)} \\
& \times\left(\begin{array}{c}
\sqrt{S c} \gamma \gamma_{1}-\sqrt{S c} \gamma\left(\gamma_{1}+\gamma_{2}+2 \gamma_{1} \gamma_{2} \eta\right) \cosh \left(L_{2} \eta\right)+ \\
\sqrt{S c} \gamma \gamma_{2} \cosh \left(2 L_{2} \eta\right)+\sqrt{\gamma-D u \operatorname{Pr} S c S r \gamma} \gamma_{1} \gamma_{2} \sinh \left(2 L_{2} \eta\right)
\end{array}\right), \\
A= & \frac{16 \pi^{6} \epsilon^{3}\left(2\left(E_{1}+E_{2}\right) \pi \cos 2 \pi(x-t)-E_{3} \sin 2 \pi(x-t)\right)^{3}}{(1+M)^{4}} .
\end{aligned}
$$

\section{Results and discussion}

The objective of this section is to analyze the behavior of parameters included in longitudinal velocity $u$, temperature $\theta$, heat transfer coefficient $Z$, mass concentration $\phi$ and stream function $\psi$. Specifically the variation of elastic tension in the membrane $\left(E_{1}\right)$, the mass per unit area $\left(E_{2}\right)$, the coefficient of viscous damping $\left(E_{3}\right)$, the Schmidt number $(S c)$, the Prandtl number $(\mathrm{Pr})$ the Soret number $(\mathrm{Sr})$, the Dufour number $(D u)$, the Biot numbers $\left(\gamma_{1}\right.$ at lower wall and $\gamma_{2}$ at upper wall), and the chemical reaction parameter $\gamma$ are considered. The results are displayed and discussed through plots.

\subsection{Velocity and temperature profiles}

This subsection is prepared to testify the effects of various emerging parameters on the velocity $u$ and temperature profile $\theta$. Figure 2 displays the effects of

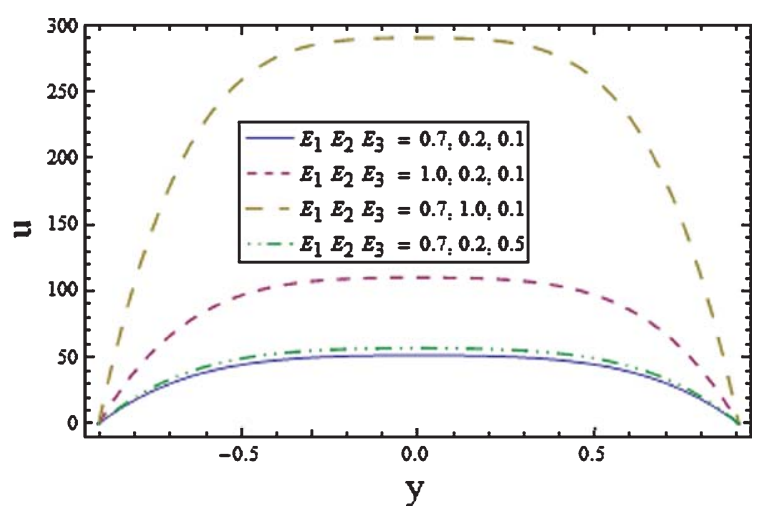

Fig. 2. Plot of longitudinal velocity $u$ for various values of the wall properties parameters with $\epsilon=0.2, x=0.02, t=0.1 M=0.5$, and $K=0.02$. wall membrane parameters $E_{1}, E_{2}$, and $E_{3}$ on velocity $u$. Graphical results show that with increase in $E_{1}$ and $E_{2}$ the velocity profile increases. Since walls are compliant and exhibits elastic behavior that offers less resistance to flow which in turn speed up the velocity. $E_{3}$ the coefficient of viscous damping demonstrates the

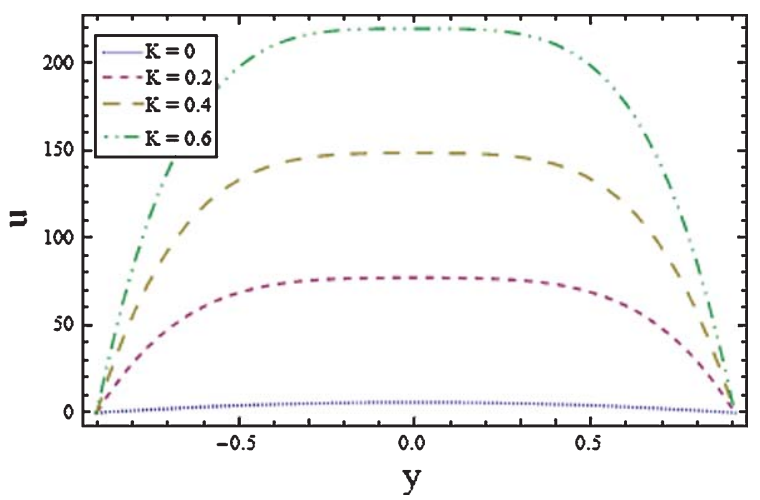

Fig. 3. Plot of longitudinal velocity $u$ for various values of the Eyring-Powell fluid parameter $K$ with $\epsilon=0.2, x=0.02, t=0.1$, $M=0.5, E_{1}=0.3, E_{2}=0.2$, and $E_{3}=0.1$.

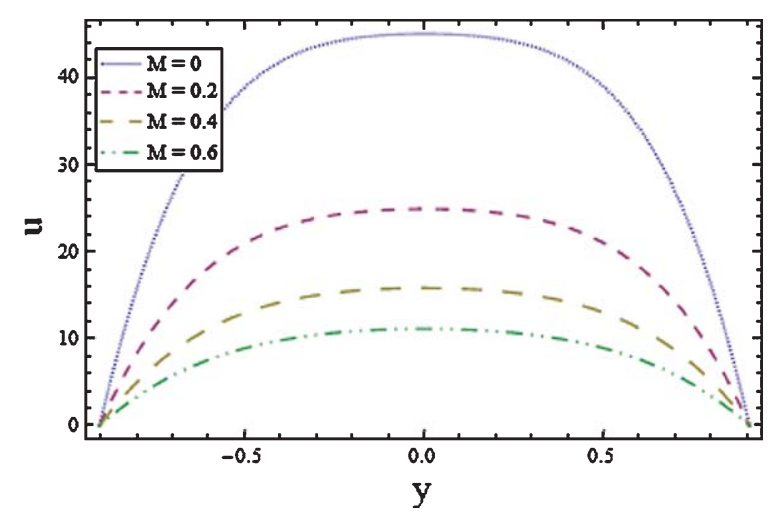

Fig. 4. Plot of longitudinal velocity $u$ for various values of the Eyring-Powell fluid parameter $M$ with $\epsilon=0.2, x=0.02, t=0.1$, $K=0.02, E_{1}=0.3, E_{2}=0.2$, and $E_{3}=0.1$. 
damping nature of the wall. It provides sufficient resistance to flow that lowers the velocity. Figures (3 and 4) show that velocity profile decreases with small $K$ while reduction in $M$ causes an increase in velocity that can be considered as characteristic of EyringPowell fluid. Figures (5 and 6) investigate the effects of Dufour number $\mathrm{Du}$ and Soret number $\mathrm{Sr}$ on the temperature profile. Graphical results indicate that with increase in the values of $D u$ and $S r$ the temperature decreases. However effects of Soret number $\mathrm{Sr}$ are more pronounced. It is apparent that an increase in $D u$ reduces the temperature of the wall which causes reduction in thermal boundary layer. An increase in $\mathrm{Sr}$ reduces the mean free temperature of fluid which results in decrease of thermal boundary. Effects of Biot numbers $\gamma_{1}$ and $\gamma_{2}$ are shown in the Figures (7 and 8). The Biot numbers are taken greater than 1 due to non-

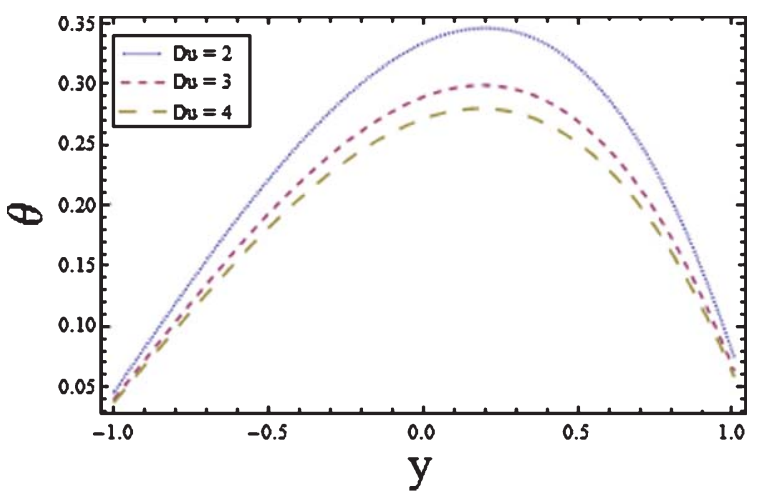

Fig. 5. Plot of the temperature $\theta$ for various values of the Dufour number $D u$ with $\epsilon=0.2, x=0.1, \gamma=-1.5, t=0.1$, $S c=0.4, S r=2, \gamma_{1}=10, P r=1.5$ and $\gamma_{2}=8$.

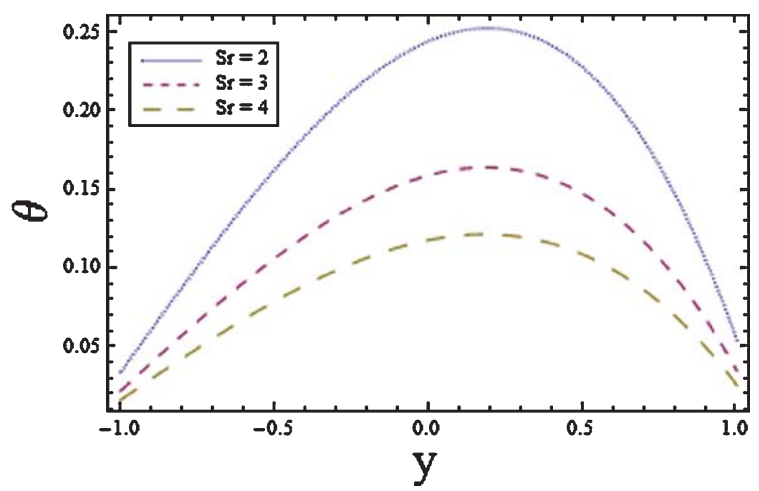

Fig. 6. Plot of the temperature $\theta$ for various values of the Soret number $S r$ with $\epsilon=0.2, x=0.1, t=0.1, S c=1, D u=2, \gamma=$ $-1.5, \gamma_{1}=10, \operatorname{Pr}=1.5$ and $\gamma_{2}=8$.

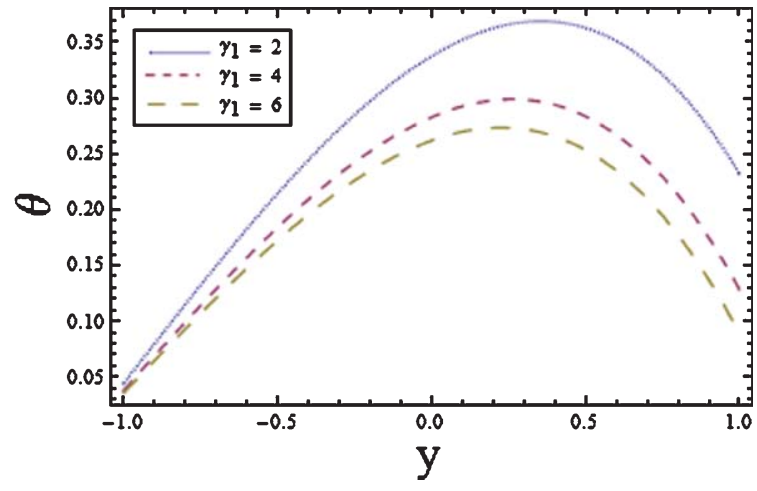

Fig. 7. Plot of the temperature $\theta$ for various values of the Biot number $\gamma_{1}$ with $\epsilon=0.2, x=0.1, t=0.1, S c=1, S r=2, D u=2$, $\gamma=-1.5, \operatorname{Pr}=1.5$ and $\gamma_{2}=8$.

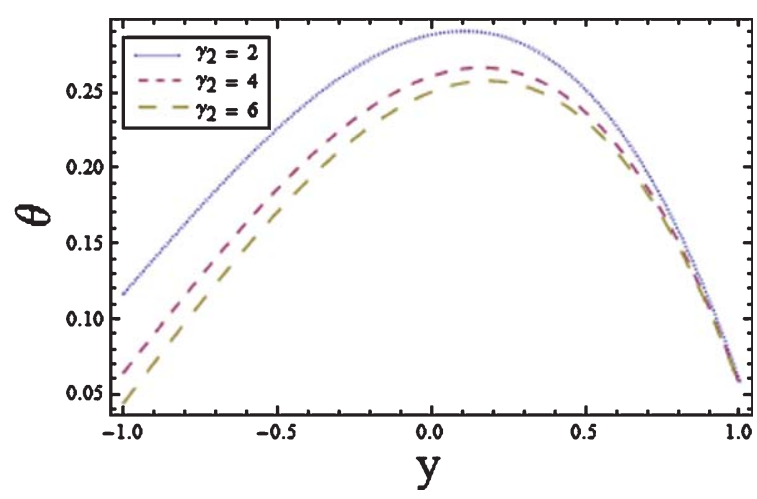

Fig. 8. Plot of the temperature $\theta$ for various values of the Biot number $\gamma_{2}$ with $\epsilon=0.2, x=0.1, t=0.1, S c=1, S r=2, D u=2$, $\gamma=-1.5, \operatorname{Pr}=1.5$ and $\gamma_{1}=10$.

uniformity of the temperature fields within the fluid. Whereas problems involving small Biot numbers are thermally simple due to the uniform temperature fields inside the fluid. Results obtained manifests decrease in temperature profile with increasing Biot numbers on the lower and upper walls. As thermal conductivity decreases with increase in Biot number justifying the temperature drop at the boundary. The ascending values of Schmidt number $S c$ on temperature distribution are depicted in Figure 9. These values show decline of thermal boundary layer. As growing values of Schmidt number $S c$ causes increase in viscosity exerting more resistance to temperature rise at the boundary. Figure 10 displays impacts of chemical reaction parameter $\gamma$ on temperature distribution. The rate of chemical reaction increases for generative chemical reaction $(\gamma<0)$ which promotes thermal boundary. Therefore temperature rise for constructive/generative 


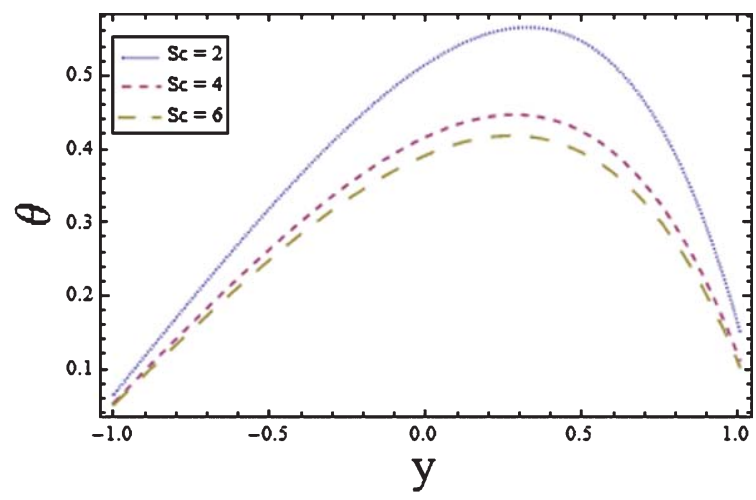

Fig. 9. Plot of the temperature $\theta$ for various values of the Schmidt number $S c$ with $\epsilon=0.2, x=0.1, t=0.1, S r=0.8, D u=0.8$, $\gamma=-1.5, \gamma_{1}=10, \operatorname{Pr}=1.5$ and $\gamma_{2}=8$.

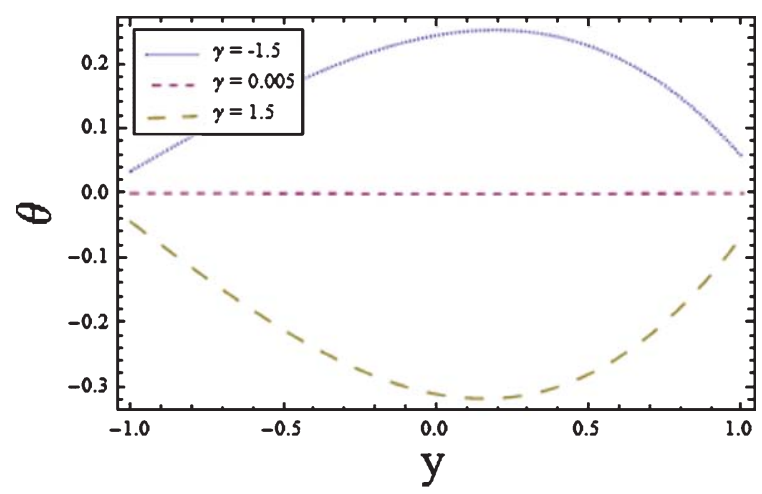

Fig. 10. Plot of the temperature $\theta$ for various values of the chemical reaction parameter $\gamma$ with $\epsilon=0.2, x=0.1, t=0.1 S c=1, S r=2$, $D u=2, \gamma_{1}=10, \operatorname{Pr}=1.5$ and $\gamma_{2}=8$.

chemical reaction is dominating than destructive chemical reaction. Also in absence of chemical reaction the temperature remains uniform. Figure 11 illustrates that temperature profile decreases with an increase in Prandtl number $P r$.

\subsection{Concentration profile}

Effects of Dufour number $\mathrm{Du}$ and Soret number $\mathrm{Sr}$ are shown in the Figures (12 and 13). Here concentration decreases when Dufour and Soret numbers are increased. Figure 14 presents the consequences of chemical reaction parameter $\gamma(\gamma>0$ for destructive reaction and $\gamma<0$ for generative reaction) on concentration profile. It is seen that concentration distribution for destructive chemical reaction is greater than generative chemical reaction. It is in view of the fact that the rate of chemical reaction increases with genera-

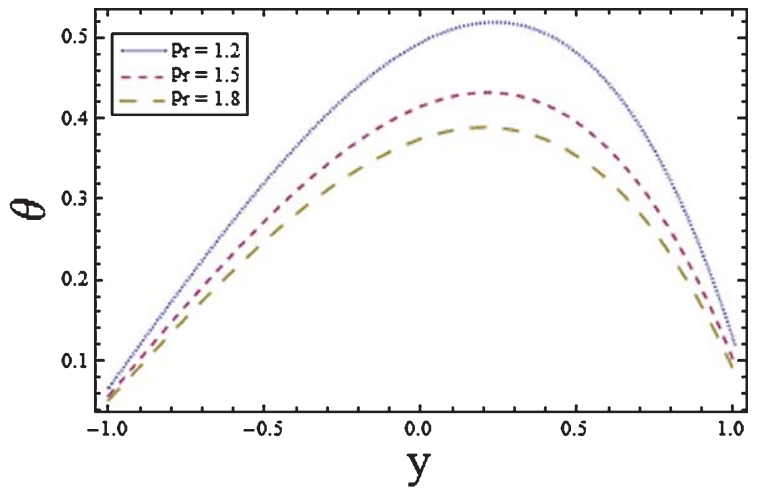

Fig. 11. Plot of the temperature $\theta$ for various values of the Prandtl number $\operatorname{Pr}$ with $\epsilon=0.2, x=0.1, t=0.1 S c=0.4, \quad S r=1.8$, $D u=1.8, \gamma_{1}=10, \gamma=-1.5$ and $\gamma_{2}=8$.

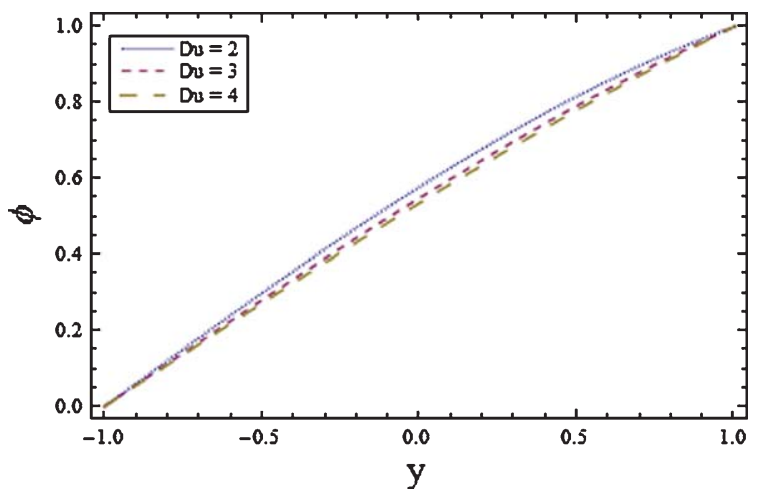

Fig. 12. Plot of concentration $\phi$ for various values of the Dufour number $D u$ with $\epsilon=0.1, x=0.1, t=0.1, S r=2, S c=2, \operatorname{Pr}=$ 1.2 and $\gamma=1.5$.

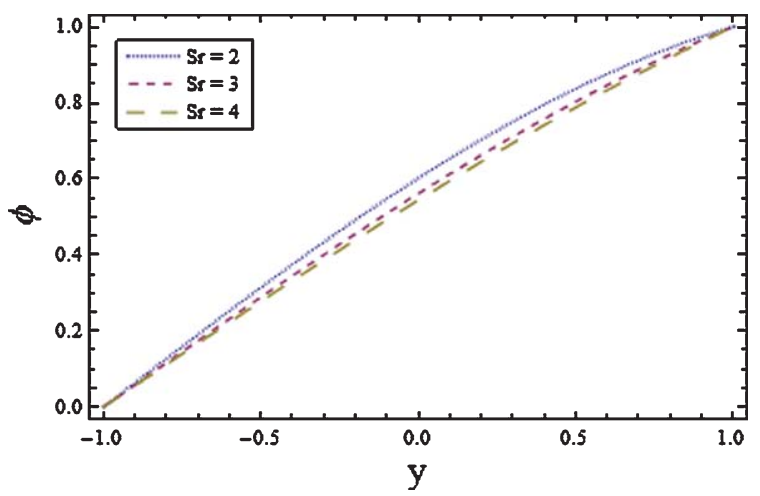

Fig. 13. Plot of concentration $\phi$ for various values of the Soret number $S r$ with $\epsilon=0.1, x=0.1, t=0.1, D u=2, S c=2, \operatorname{Pr}=$ 1.2 and $\gamma=1.5$. 


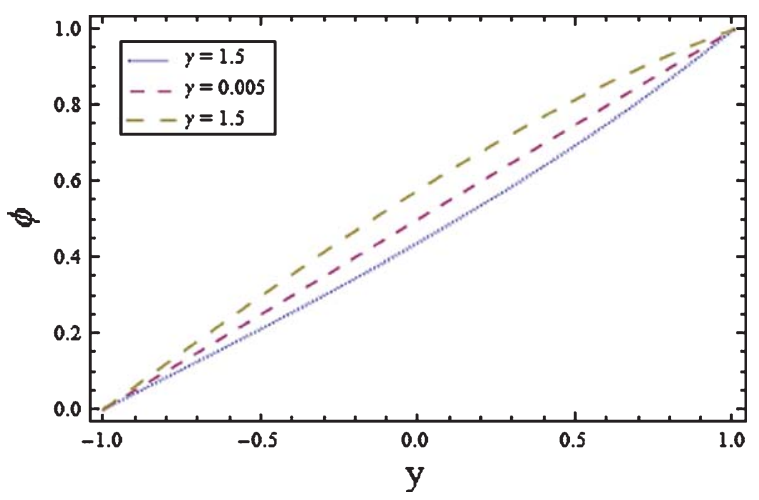

Fig. 14. Plot of concentration $\phi$ for various values of the Chemical reaction parameter $\gamma$ with $\epsilon=0.1, x=0.1, t=0.1, S r=2, S c=$ 2, $P r=1.2$ and $D u=2$.

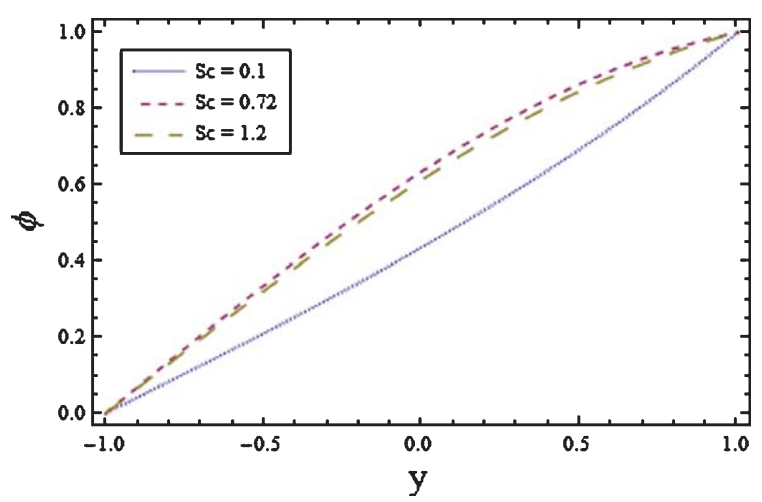

Fig. 15. Plot of concentration $\phi$ for various values of the Schmidt number $S c$ with $\epsilon=0.1, x=0.1, t=0.1, S r=2, \gamma=1.5, \operatorname{Pr}=$ 1.2 and $D u=2$.

tive chemical reaction which lessens the concentration profile. For larger values of Schmidt number $S c$, the molecular diffusion decreases that dominates intermolecular forces between molecules and hence there is increase in concentration (see Figure 15). Increase in values of Prandtl number $\operatorname{Pr}$ causes decrease of concentration (see Figure 16). It is in view of the fact that an increase in $P r$ enhances viscosity of fluid which in turns lessen the concentration distribution.

\subsection{Heat transfer coefficient}

The aim of this subsection is to examine the impacts of various fluid parameters on the heat transfer coefficient $Z$. From Figures $(17-22)$ it is noticed that the heat transfer coefficient $Z$ has dual behavior for increasing Soret number $S r$, Dufour number $\mathrm{Du}$, Biot numbers $\gamma_{1}, \gamma_{2}$ Schmidt number $S c$ and Prandtl number $P r$.

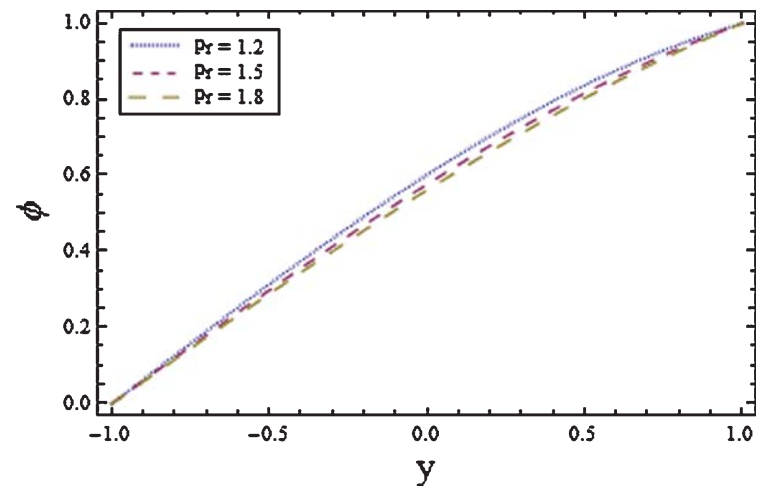

Fig. 16. Plot of concentration $\phi$ for various values of the Prandtl number $\operatorname{Pr}$ with $\epsilon=0.1, x=0.1, t=0.1, S r=2, \gamma=1.5, S c=2$ and $D u=2$.

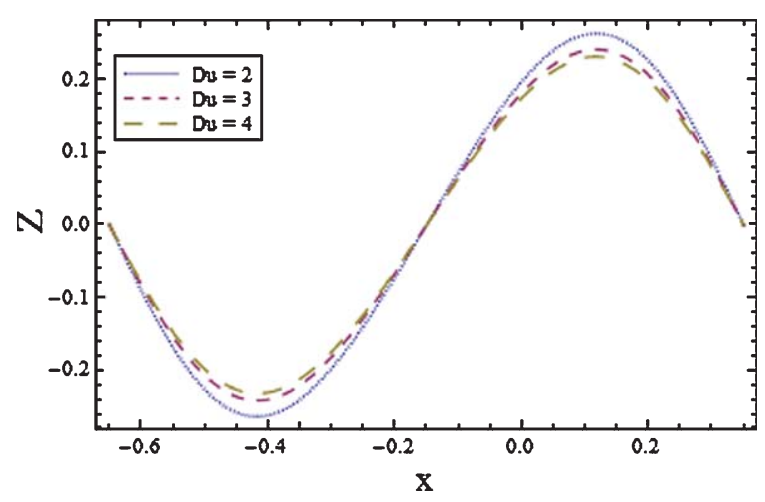

Fig. 17. Plot of heat transfer coefficient $Z$ for various values of the Dufour number $D u$ with $\epsilon=0.1, t=0.1, S c=1, S r=2, \gamma=1$, $\gamma_{1}=10, \operatorname{Pr}=1.5$ and $\gamma_{2}=8$.

Figure 17 shows enhancing $D u$ which favours heat transfer for smaller values $(x<-0.2)$ while greater values $(x>-0.1)$ of $D u$ reduce heat transfer distribution. Figure 18 shows oscillating behavior, heat transfer distribution decreases with ascending values of $\mathrm{Sr}$ when $x<0.4$, and it increases when $x>0.4$. In Figure 19 it is noticed that ascending values of Biot numbers $\gamma_{1}$ develops heat transfer coefficient positively for $(x>-0.1)$ (since Biot number is directly related to heat transfer coefficient of fluid) while it has opposite impact in a region where $(x<-0.2)$. It may be considered that in a region $x<-0.2$, decrease in thermal conduction with ascending values of $\gamma_{1}$ exceeds heat transfer of fluid resulting decrease of heat transfer distribution in this region. In case of $\gamma_{2}$ the situation is reversed and therefore results are opposite (Figure 20). Also Schmidt number exhibits dual (oscillatory) behavior similar to that of Dufour number in Figure 


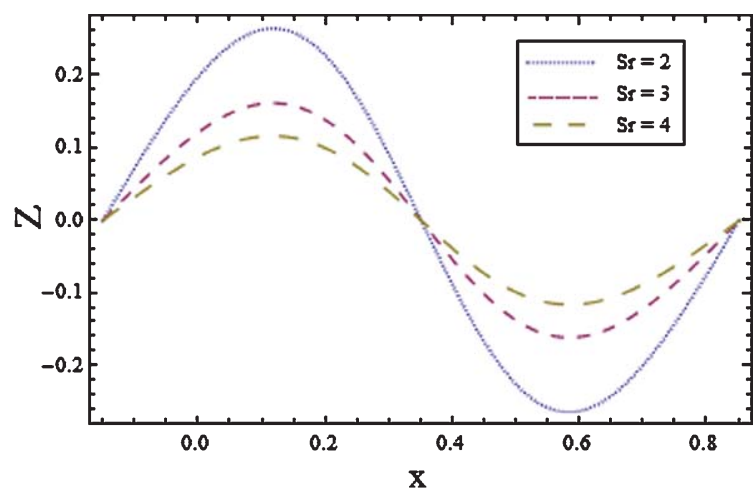

Fig. 18. Plot of heat transfer coefficient $Z$ for various values of the Soret number $S r$ with $\epsilon=0.1, t=0.1, S c=1, D u=2, \gamma=1$, $\gamma_{1}=10, \operatorname{Pr}=1.5$ and $\gamma_{2}=8$.

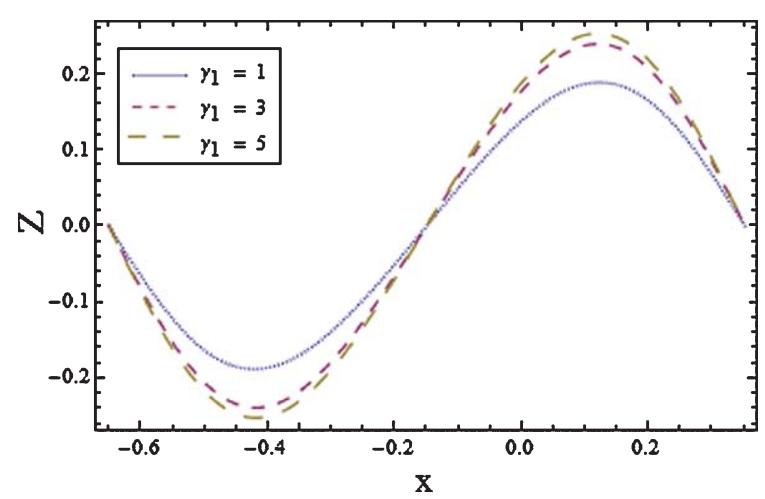

Fig. 19. Plot of heat transfer coefficient $Z$ for various values of the Biot number $\gamma_{1}$ with $\epsilon=0.1, t=0.1, S c=1, D u=2, S r=2$, $\gamma=1, \operatorname{Pr}=1.5$ and $\gamma_{2}=8$.

21. The effect of Prandtl number $\operatorname{Pr}$ on heat transfer is similar to that obtained for $D u$ (see Figures 17 and 22).

\subsection{Trapping}

An influential aspect of fluid called trapping assigns to closed and circulating streamlines. This closed internally circulating streamline is called bolus and it moves along with the peristaltic wave. Figures $(23-25)$ displays the generation of trapped bolus with the variation of wall parameters $E_{1}, E_{2}$ and $E_{3}$. Figures 23 ( $a, b$ and $c$ ) show that the size of trapped bolus decreases with increase in $E_{1}$. Figures $24(a, b$ and $c$ ) show the decrease of bolus with $E_{2}$ whereas Figures 25 ( $a, b$ and $c$ ) illustrate that an increase in $E_{3}$ has no significant change in the size of bolus. Figures $26(a, b$ and $c)$ depicts that the size of trapped bolus reduces for larger material

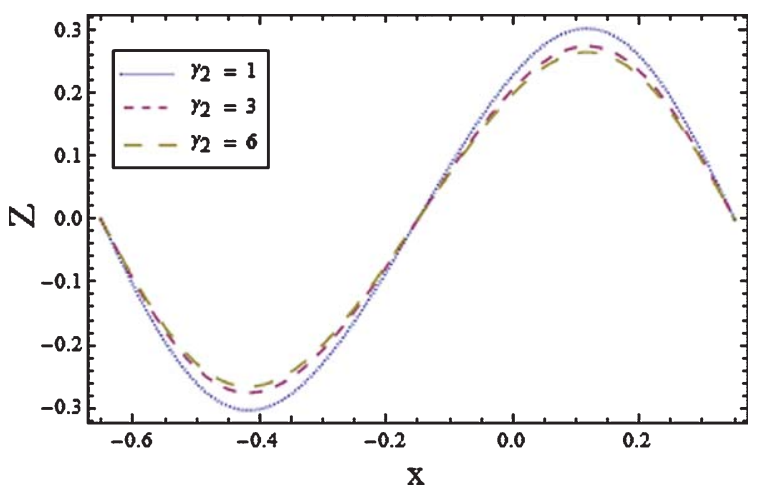

Fig. 20. Plot of heat transfer coefficient $Z$ for various values of the Biot number $\gamma_{2}$ with $\epsilon=0.1, t=0.1, S c=1, D u=2, S r=2$, $\gamma=1, \operatorname{Pr}=1.5$ and $\gamma_{1}=10$.

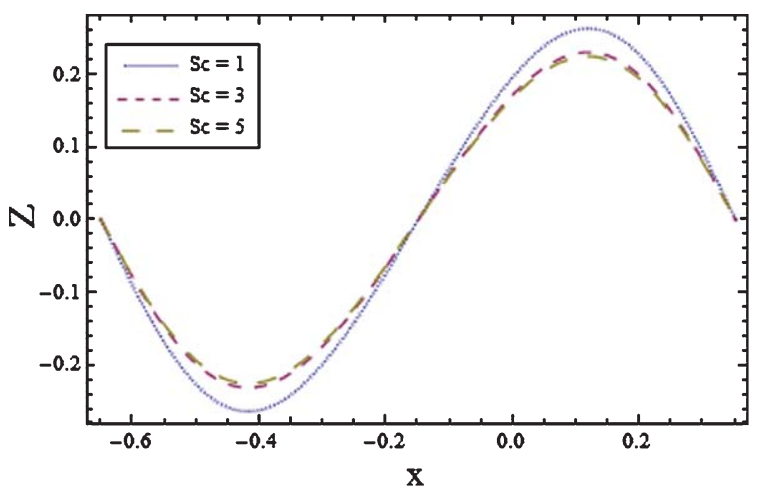

Fig. 21. Plot of heat transfer coefficient $Z$ for various values of the Schmidt number $S c$ with $\epsilon=0.1, t=0.1, S r=2, D u=2, \gamma=1$, $\gamma_{1}=10, \operatorname{Pr}=1.5$ and $\gamma_{2}=8$.

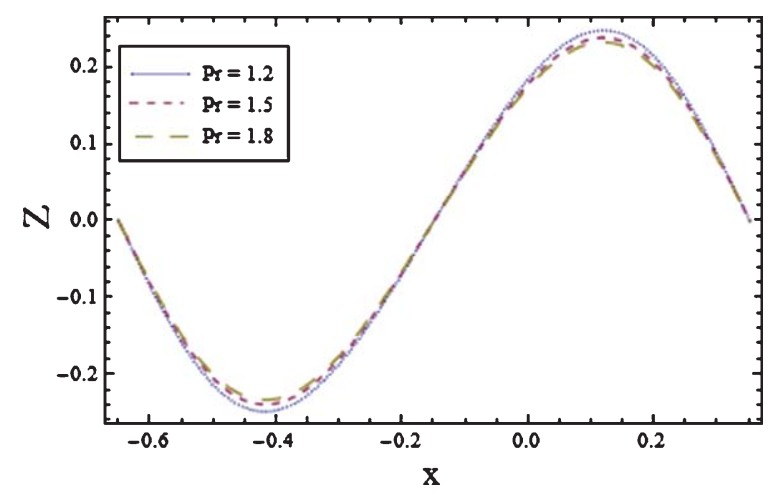

Fig. 22. Plot of heat transfer coefficient $Z$ for various values of the Prandtl number $\operatorname{Pr}$ with $\epsilon=0.1, t=0.1, S r=2, D u=2, \gamma=1$, $\gamma_{1}=10, S c=2$ and $\gamma_{2}=8$.

fluid parameter $K$. Figures $27(a, b$ and $c$ ) are drawn to examine the behavior of fluid parameter $M$. Increasing $M$ lowers the size of trapped bolus. Additionally in 

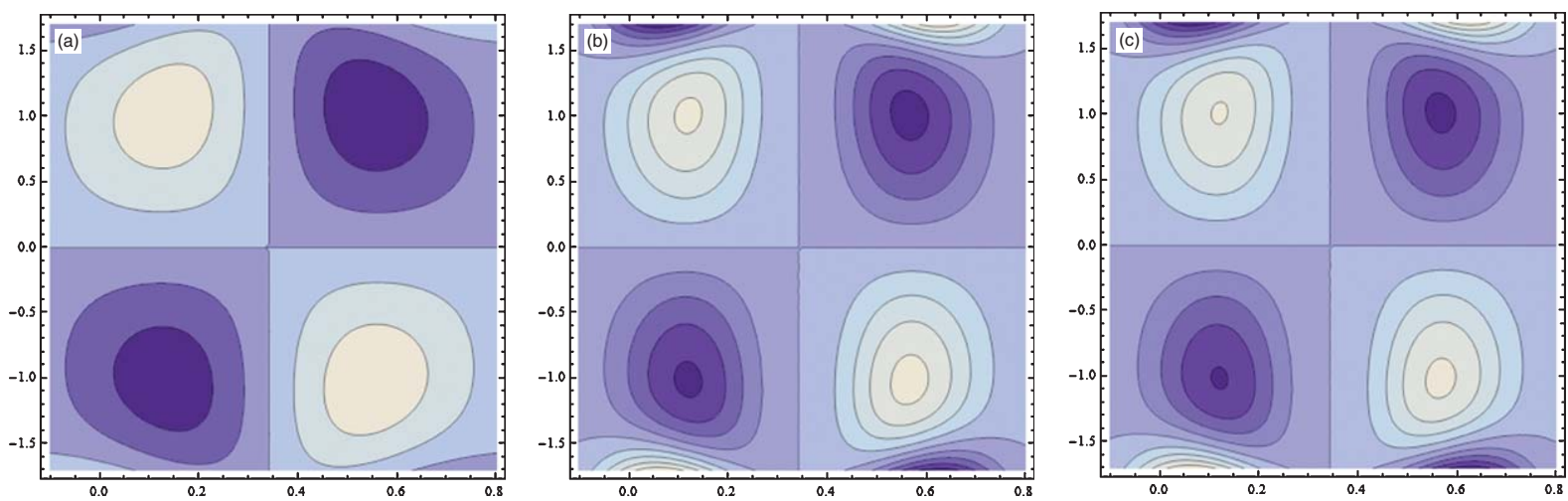

Fig. 23. Streamlines for $E_{2}=0.1 . E_{3}=0.1, \epsilon=0.1, t=0.1, M=0.5, K=0.2$, with different $E_{1}$ (a) $E_{1}=0.2$, (b) $E_{1}=0.27$. (c) $E_{1}=0.3$.
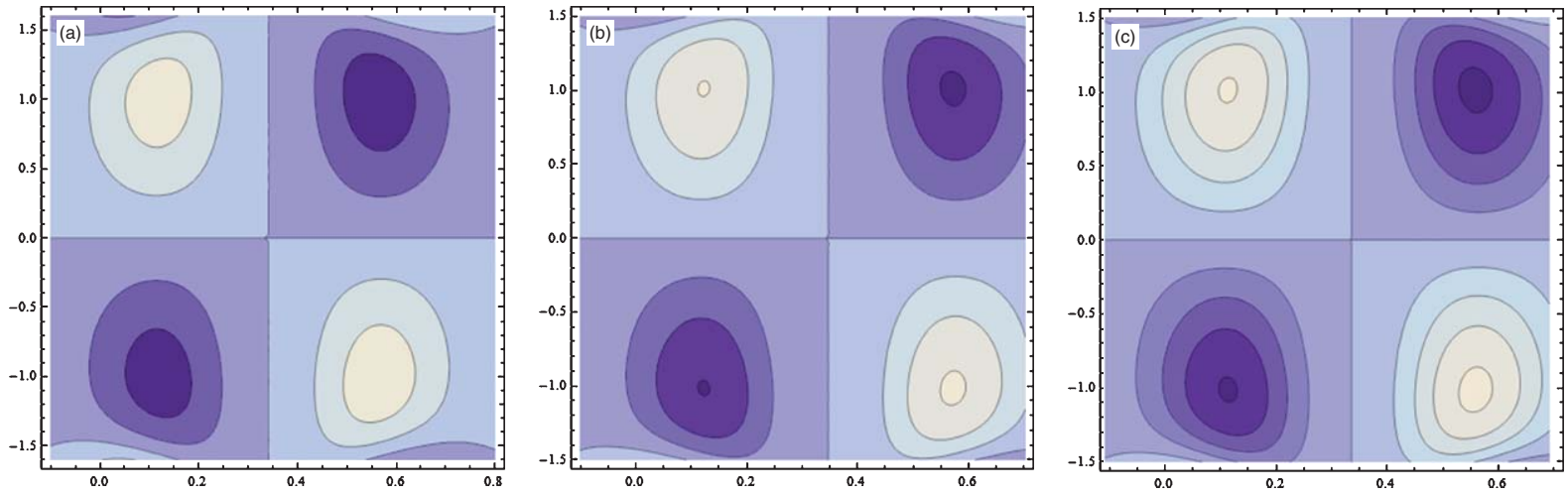

Fig. 24. Streamlines for $E_{1}=0.2, E_{3}=0.1, \epsilon=0.1, t=0.1, M=0.5, K=0.2$, with different $E_{2}$ (a) $E_{2}=0.5$, (b) $E_{2}=0.4$, (c) $E_{2}=0.2$.
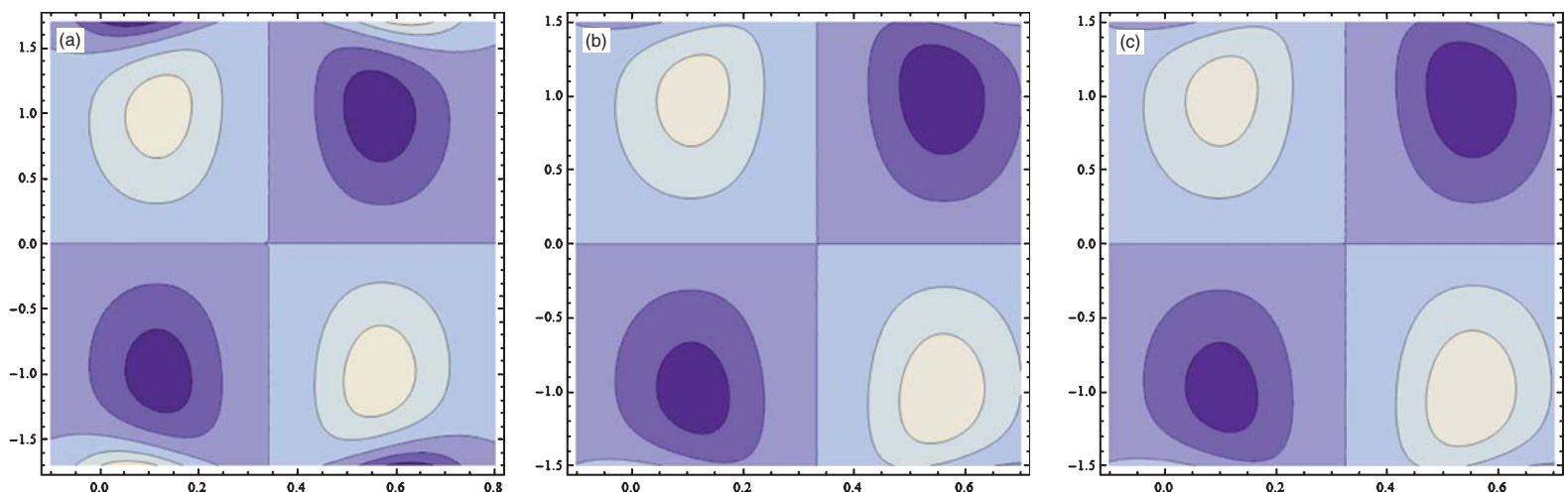

Fig. 25. Streamlines for $E_{1}=0.2, E_{2}=0.1, \epsilon=0.1, t=0.1, M=0.5, K=0.2$, with different $E_{3}$ (a) $E_{3}=0.1$, (b) $E_{3}=0.2$, (c) $E_{3}=0.3$.

absence of Eyring-Powell fluid parameters the trapped bolus is similar to that of viscous fluid.

\section{Concluding remarks}

Here we analyzed the peristaltic flow of EyringPowell fluid in a symmetric channel with compliant walls and convective conditions. Analysis has been carried out in the presence of Soret and Dufour numbers. The main observations are listed below:

- The velocity field gives opposite results with increasing values of Eyring-Powell fluid parameters $M$ and $K$. 

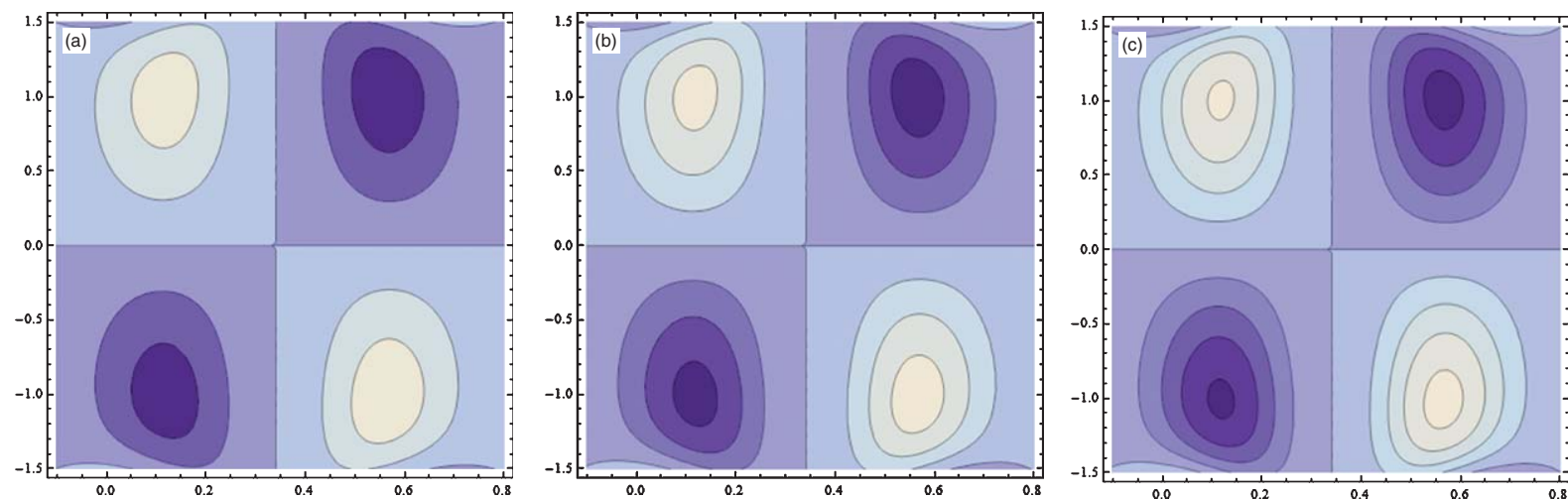

Fig. 26. Streamlines for $E_{1}=0.2, E_{2}=0.1, E_{3}=0.1, \epsilon=0.1, t=0.1, M=0.5$, with different $K$ (a) $K=0.2$, (b) $K=0.3$, (c) $K=0.4$
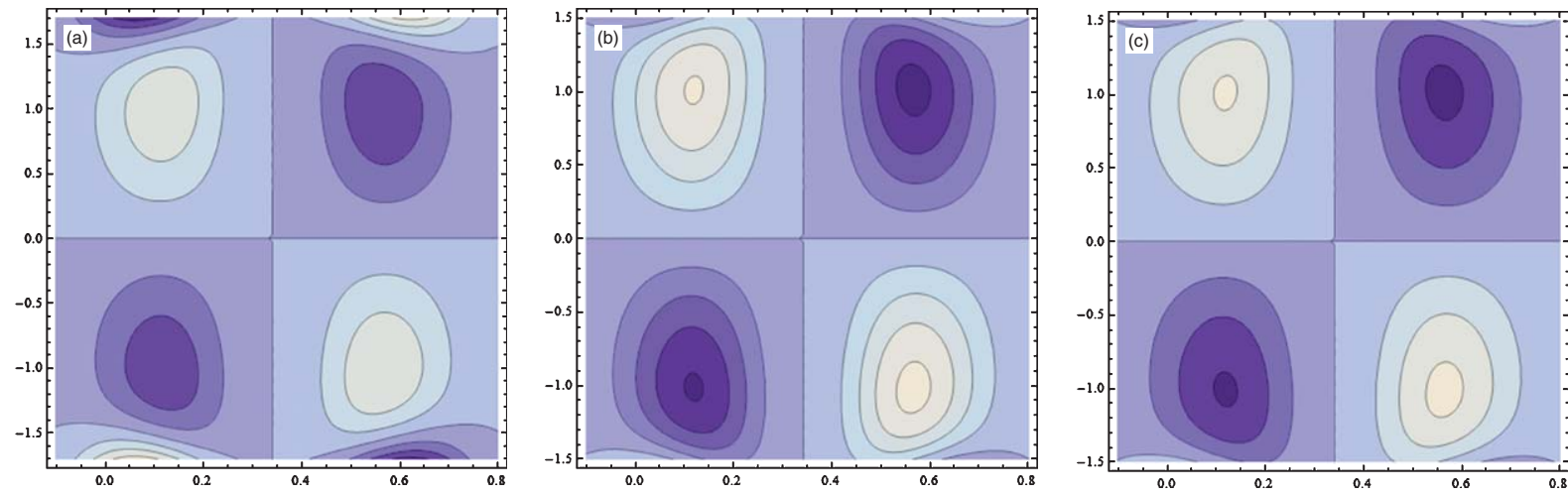

Fig. 27. Streamlines for $E_{1}=0.2, E_{2}=0.1, E_{3}=0.1, \epsilon=0.1, t=0.1, K=0.2$, with different $M$ (a) $M=0.2$, (b) $M=0.3$, (c) $M=0.4$.

- Similar behavior of temperature and concentration profiles is observed for increasing values of Soret and Dufour parameters.

- Temperature is decreasing function of Biot numbers, which shows convective cooling near the channel walls. Further the Biot numbers have dual effect on heat transfer coefficient.

- The results for the prescribed surface temperature (PST) are deduced as a special case of the present analysis when $\gamma_{1} \rightarrow \infty$ and $\gamma_{2} \rightarrow \infty$.

- Increasing Schmidt number shows reduction of temperature distribution and enhancement of concentration profile.

- The size of trapped bolus decreases through increase of Eyring-Powell fluid parameters and elasticity parameters.

\section{References}

[1] M. Jamil and C. Fetecau, Some exact solutions for rotating flows of a generalized Burgers' fluid in cylindrical domains, J Non-Newtonian Fluid Mech 165 (2010), 1700-1712.
[2] M. Jamil, C. Fetecau and M. Imran, Unsteady helical flows of Oldroyd-B fluids, Commun Nonlinear Sci Numerical Simulat 16 (2011), 1378-1386.

[3] M. Turkyilmazoglu and I. Pop, Exact analytical solutions for the flow and heat transfer near the stagnation point on a stretching/shrinking sheet in a Jeffrey fluid, Int $J$ Heat and Mass Transfer 57 (2013), 82-88.

[4] M.M. Rashidi, S. Abelman and N. Freidooni Mehr, Entropy generation in steady MHD flow due to a rotating porous disk in a nanofluid, Int J Heat and Mass Transfer 62 (2013), 515-525.

[5] T. Hayat, M. Awais and S. Asghar, Radiative effects in a three dimensional flow of MHD Eyring-Powell fluid, J Egyptian Math Society 21 (2013), 379-384.

[6] S. Nadeem, A. Riaz and R. Ellahi, Peristaltic flow of a Jeffrey fluid in a rectangular duct having compliant walls, Chem Industry \& Chem Eng Quarterly 19 (2013), 399-409.

[7] Y. Abd. Elmaboud and K.S. Mekheimer, Non-linear peristaltic transport of a secondorder fluid through a porous medium, Appl Math Modelling 35 (2011), 2695-2710.

[8] D. Tripathi, S.K. Pandey and S. Das, Peristaltic flow of viscoelastic fluid with fractional Maxwell model through a channel, Appl Math and Comput 215 (2010), 3645-3654.

[9] N. Ali, T. Hayat and S. Asghar, Peristaltic flow of a Maxwell fluid in a channel with compliant walls, Chaos Solitons and Fractals 39 (2009), 407-416.

[10] S.K. Pandey and M.K. Chaube, Peristaltic flow of a micropolar fluid through a porous medium in the presence of an 
external magnetic field, Commun Nonlinear Sci Numerical Simulat 16 (2011), 3591-3601.

[11] M. Mustafa, S. Hina, T. Hayat and A. Alsaedi, Influence of wall properties on the peristaltic flow of a nanofluid: Analytic and numerical solutions, Int J Heat and Mass Transfer 55 (2012), 4871-4877.

[12] R. Ellahi, A. Riaz and S. Nadeem, Three dimensional peristaltic flow of Williamson fluid in a rectangular duct, Indian J Phys 87 (2013), 1275-1281.

[13] T. Hayat, S. Hina, M. Mustafa and S. Abbasbandy, Numerical investigation on mixed convective peristaltic flow on fourth grade fluid with Dufour and Soret effects, J Taiwan Institute of Chemical Engineers (2013). DOI: org/10.1016/j.jtice

[14] K.S. Mekheimer, N. Saleem, T. Hayat and A.A. Hendi, Simultaneous effects of induced magnetic field and heat and mass transfer on the peristaltic motion of second-order fluid in a channel, Int J Numerical Methods 70 (2011), 342-358.

[15] K.S. Mekheimer, S.Z.A. Husseny and Y. Abd. Elmaboud, Effects of heat transfer and space porosity on peristaltic flow in a vertical asymmetric channel, Numerical Methods for P. D. Eqs 26 (2010), 747-770.

[16] S. Srinivas and M. Kothandapani, The influence of heat and mass transfer on MHD peristaltic flow through a porous space with compliant walls, Appl Math and Comput 213 (2009), 197-208.

[17] S. Srinivas, R. Gayathri and M. Kothandapani, Mixed convective heat and mass transfer in an asymmetric channel with peristalsis, Commun Nonlinear Sci Numerical Simulat 16 (2011), 1845-1862.

[18] M. Saleem and A. Haider, Heat and mass transfer on the peristaltic transport of non-Newtonian fluid with creeping flow, Int J Heat and Mass Transfer 68 (2014), 514-526.

[19] R. Ellahi, M. Mubashir Bhatti and K. Vafai, Effects of heat and mass transfer on peristaltic flow in a non-uniform rectangular duct, Int J Heat and Mass Transfer 71 (2014), 706-719.
[20] T. Hayat, S. Hina, A.A. Hendi and S. Asghar, Influence of compliant walls on peristaltic motion with heat/mass transfer and chemical reaction, Int J Heat and Mass Transfer $\mathbf{5 5}$ (2012), 3386-3394.

[21] S. Hina, T. Hayat and A. Alsaedi, Heat and mass transfer effects on the peristaltic flow of Johnson-Segalman fluid in a curved channel with compliant walls, Int J Heat and Mass Transfer 55 (2012), 3511-3521.

[22] R. Ellahi, A. Riaz, S. Nadeem and M. Mushtaq, Series solutions of magnetohydrodynamic peristaltic flow of a Jeffrey fluid in eccentric cylinders, J Appl Math Info Sci 7 (2013), 1441-1449.

[23] M. Sheikholeslami, M. Gorji Bandpy, R. Ellahi, M. Hassan and S. Soleimani, Effects of MHD on Cu-water nanofluid flow and heat transfer by means of CVFEM, J Mag Mag Materials 349 (2014), 188-200.

[24] M. Sheikholeslami, R. Ellahi, H.R. Ashorynejad, G. Domairry and T. Hayat, Effects of heat transfer in flow of nanofluids over a permeable stretching wall in a porous medium, J Comp Theor Nanosci 11 (2014), 486-496.

[25] A.Zeeshan and R. Ellahi, Series solutions for nonlinear partial differential equations with slip boundary conditions for nonNewtonian MHD fluid in porous space, J Appl Math Info Sci 7 (2013), 253-261.

[26] T. Hayat, Z. Iqbal, M. Qasim and S. Obaidat, Steady flow of an Eyring Powell fluid over a moving surface with convective boundary conditions, Int J Heat and Mass Transfer 55 (2012), 1817-1822.

[27] A.V. Rosca and I. Pop, Flow and heat transfer of Powell-Eyring fluid over a shrinking surface in a parallel free stream, Int J Heat and Mass Transfer 71 (2014), 321-327.

[28] M. Jalil, S. Asghar and S.M. Imran, Self similar solutions for the flow and heat transfer of Powell-Eyring fluid over a moving surface in a parallel free stream, Int J Heat and Mass Transfer 65 (2013), 73-79. 

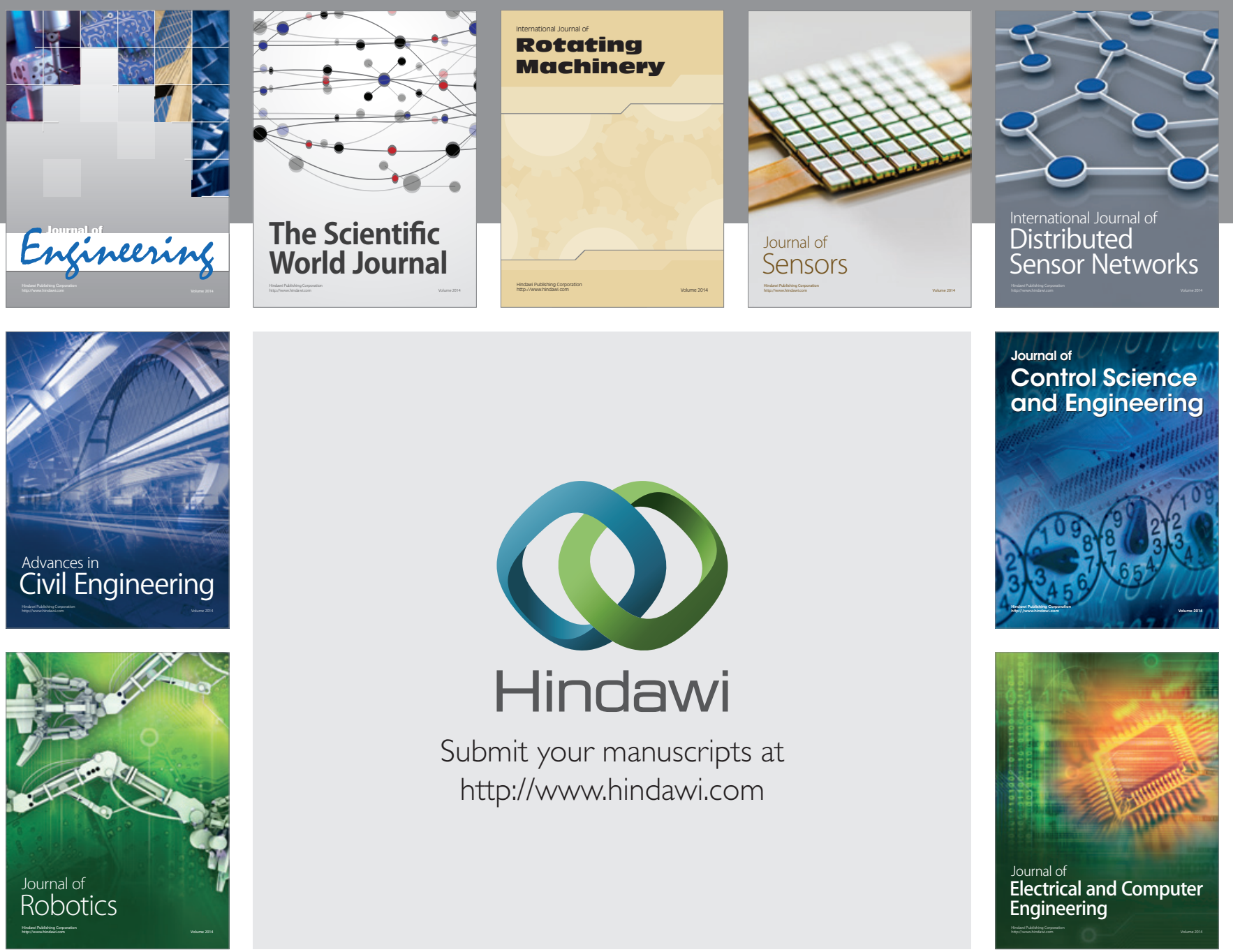

Submit your manuscripts at

http://www.hindawi.com
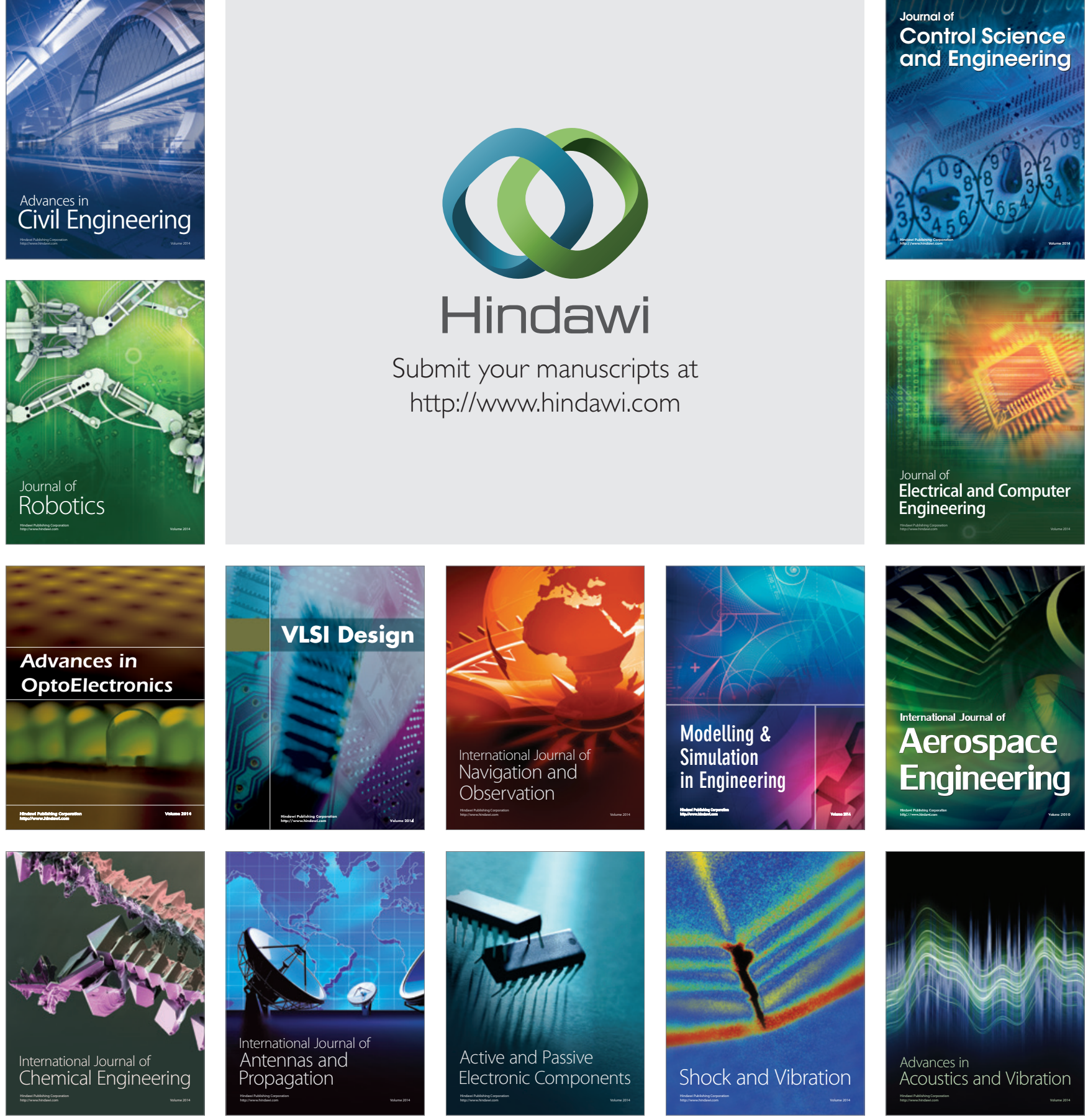